\title{
Urban population agglomeration in view of complex ecological niche: A case study on Chinese prefecture cities
}

\author{
Baolong Han ${ }^{\mathrm{a}}$, Rusong Wang ${ }^{\mathrm{a}, *}$, Yu Tao ${ }^{\mathrm{a}}, \mathrm{Hui}$ Gao ${ }^{\mathrm{a}, \mathrm{b}}$ \\ a State Key Laboratory of Urban and Regional Ecology, Research Centre for Eco-Environmental Sciences, Chinese Academy of Sciences, Shuangqing Road 18, \\ Haidian District, Beijing 100085, China \\ ${ }^{\mathrm{b}}$ College of Urban and Environmental Science, Xinyang Normal University, Xinyang 46400, Henan, China
}

\section{A R T I C L E I N F O}

\section{Article history:}

Received 14 January 2014

Received in revised form 31 July 2014

Accepted 1 August 2014

\section{Keywords:}

Urban population agglomeration

Complex ecological niche

Environmental ecological niche

Social ecological niche

Economic ecological niche

\begin{abstract}
A B S T R A C T
Population agglomeration of a particular city provides evidence of its urbanization quality, as well as its appeal for immigration from rural areas to developed areas. The attractive factors for population agglomeration in urban areas are analyzed using a new perspective of the complex ecological niche (CEN). The CEN involves three groups of key elements: natural environment, economic flow and social culture. An indicator system was designed for the urban population CEN with three sub-CENs as second-level indicators, i.e., the environmental ecological niche, the social ecological niche and the economic ecological niche, each of which has five third-level measurable indicators. Another indicator was designed for evaluating the balance degree among the three sub-CENs. A case study of 220 Chinese cities during the years 2000-2011 was performed using the proposed indicators. The sub-CENs of this group of Chinese cities were positively correlated with the state of development of the cities. But the significance of the correlation was lower for the environmental ecological niche which changes less than the other two sub-CENs. The association between the three sub-CENs weakened most cities in south-eastern China, compared to cities elsewhere. Finally, analysis of the relationship between urban population agglomeration and the environmental ecological niche revealed by partial correlation analysis and regression analysis implied that there is a threshold in the development process of the environmental ecological niche. That threshold differentiates the negative and positive impacts upon urban agglomeration. Most Chinese cities are still in the negative phase.
\end{abstract}

(c) 2014 Elsevier Ltd. All rights reserved.

\section{Introduction}

In both global mega-cities and small local cities urban population growth is coupled to urban development (Fujishima, 2013; Tabuchi, 2014). Population is both the labor force for industry and the consumer of the resources that a city needs, such as food, water, coal, wood, oil and building materials. Population dynamics have a great influence on urban development, according to the endogenous economic growth theory, population play the most important role to the city's economic increase (Grafeneder-Weissteiner and Prettner, 2013; Prettner, 2013). Increasing or controlling the magnitude of the urban population is always a key issue for the sustainable development of any city.

\footnotetext{
* Corresponding author. Tel.: +86 10 62943807; fax: +86 1062943807.

E-mail addresses: baronhan@gmail.com (B. Han), wangrs@mail.rcees.ac.cn (R. Wang), george881126@126.com (Y. Tao), gaohui8010@163.com (H. Gao).
}

The factors influencing urban population changes can be grouped into three terms: natural environment, social environment and economic environment (Wang, 2007; Wang et al., 2011a). These three terms are also often considered when evaluating sustainable development (Munier, 2011; Tanguay et al., 2010; Theodoridou et al., 2012). Tabuchi (2013) recognizes that safety, convenience and job opportunities are the main reasons for rural to urban migration. He found a unidirectional influence of the economy on population change. $\mathrm{Wu}$ (2008) found the labor market and urban wetland amount to be significant driving forces of population distribution in a city. An (2012) and Gaigné et al. (2012) thought that it was necessary to consider the coupled relationship between population and those factors. Sato and Yamamoto (2005) announced that the changes in the urban population scale and the changes in the urban population density differ. The former is significant with a city's economic scale, which is what these authors considered in the city development evaluation jobs. Population density however has a closer relationship with social development, environmental conditions and other factors of more concern to individuals (Tan 
et al., 2008). Shoshany and Goldshlager (2002) and Smailes et al. (2002) implied that those factors which influence individuals more could be seen as some kind of niche for citizens.

From the literature cited above, it may be concluded that (1) little research has looked at the city as a whole unit and has compared cities to investigate the different factors that attracted their populations; (2) the difference between population scale and density implies that there is a need to analyze urban population agglomeration changes from an individual's standpoint, which suggests that a human ecological niche analysis needs to be conducted for urban citizens. And these suggest that we can classify those factors into three terms which were named complex ecological niche (CEN) and its three sub-CENs.

Urban population complex ecological niche differs from the human ecological niche that measures the conditions humans need to survive (Banks et al., 2008). The CEN for urban population agglomeration (UPA) expresses the characteristics of the city that has impact upon people's decisions on their immigration. Imagine the CEN as an intersection space on a multidimensional coordinate axis, with each factor as one coordinate axis; that is to say, one city's actual CEN is just a point with the city's different features as its multidimensional coordinate. Furthermore, the CEN for UPA for a country means the space range constituted by all the cities' CEN points. Furthermore, this research program not only suggests an original view from CEN of UPA but also develops an original indicator system for CEN based on the demands of individuals, rather than the entire city's development scale, as previous scholars have done. There are three sub-CENs in the CEN indicator system which are analyzed in terms of the changes within them and their relationships with UPA over the past 12 years.

Following the introduction, the second section describes the study sites and the indicators for CEN and sub-CENs. Then it sets out the methods to calculate those indicators, and simulate to the relationship between UPA and CEN, and sets out a way of evaluating the influence of CEN and its changes. Results presented in the third section of this paper reveal the complexity of CEN-UPA issue, and analyze the driving forces from sub-CENs to UPA. They then explore the changes of sub-CENs in China during the past 11 years. In the fourth section, previous sections are explained and discussed in terms of the relationship between natural environmental ecological niche and UPA, with some issues and guidance for future. The fifth section summarizes the whole paper, and recommends some policy initiatives stemming from the work reported here.

\section{Method}

\subsection{Study sites}

China is the largest developing country in the world undergoing fast economic and population increase as well as urbanization; China's economy has been ranked second in the world (Zhang and Zhao, 2003). By the end of 2011, China's population have increased by 2.48 times compared with that in 1949 (the founding of New China); the urbanization rate exceeded $50 \%$ which is mainly due to the fast urbanization during 1999-2009 (Gu et al., 2012) (Fig. 1).

China and 220 Chinese prefecture cities were chosen as the research objects for three reasons. First, both the types of labor and cities are numerous in China, which gives people a wide choice to migrate freely. Second, China is experiencing a rapid urbanization which has led to internal imbalances in its development level with respect to environmental, economic, and social aspects (Yu and Wen, 2010), making the affecting factors for urban population agglomeration more effective. Third, as the world's most populous



Fig. 1. Change in the Chinese population and urbanization since the founding of China in 1949.

country, there are significant practical reasons for analyzing the CEN for Chinese UPA and for making policy suggestions for China. There are more than 600 cities in China, and 285 of them are prefecture cities. 220 cities were selected and there is no missing data during the years 2000-2011 from all the prefecture cities. All the original data used in the complexity analysis and indicator calculation were obtained from the China City Statistical Yearbook (from 2000 to 2011).

\subsection{Urban population CEN indicators}

Complex ecosystem theory says that the key elements affecting the development of a city can be summarized in three aspects (Wang et al., 2011b; Zhang et al., 2006): natural environment, economic flow and social culture (Wang, 2007; Wang et al., 2011a). Developing this theme, CEN for UPA can be made up of three sub-CENs: natural environmental ecological niche (NCEN), social ecological niche (SCEN), and economic ecological niche (ECEN) (Fig. 2). As these sub-CENs are all potential indicators, five measurable indicators were chosen for each of them. For NCEN, five aspects required to be considered are air conditions, water conditions, green land, environmental diversity and residential environment. Each of them was measured with observable indicators (Table 1). For SCEN, education guarantee, health guarantee, population quality, cultural communication, and entertainment sets were the most important factors. For ECEN, work income, production efficiency, economic openness, financial viability and infrastructure construction expressed the most important factor affecting the choices made by individuals. This table of indicators shows that the CEN indicator system cares more about individual well-being, rather than the total development level of the city. This is the obvious difference compared with the traditional city sustainable indicator system, which contain indicators at several scales.

At the beginning, this paper analyzed the relationship among urban development, urban population agglomeration (UPA) and urban population scale (UPS). The measure indicator for UPA was population density and was the sum population for UPS.

\subsection{Sub-CENs calculation}

All the 15 third-level indicators had observant variables to measure them, and three second-level indicators (three sub-CENs) were abstracted from those third level indicators. Information is lost in reducing the indicators from 15 dimensions to three dimensions. To minimize this information loss, an entropy weighting method is used to calculate each of the three sub-CENs.

Before the calculation, all the data were standardized to eliminate the influence of units and scales using the following procedure.

For the sample sid in a sample collection that has $n$ samples, define $X_{\text {sid }}$ as sid's value under indicator $X . X \in\left(N_{1}, N_{2}, \ldots N_{5}, S, S_{2}\right.$, 




Urban complex-ecosystem

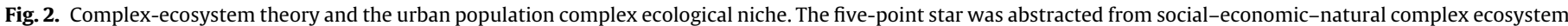
(Wang et al., 2011a)

$\left.\ldots S_{5}, E_{1}, E_{2}, \ldots E_{5}\right)$ define $X_{\text {sid }}^{\prime}$ as the standardized $X_{\text {sid }}: X_{\text {sid }}^{\prime} \in[0,1]$. Define $\operatorname{MIN}(X)$ and $\operatorname{MAX}(X)$ :

$\operatorname{MIN}(X)=\operatorname{MIN}\left(X_{1}, X_{2}, \ldots, X_{\text {sid }}, \ldots, X_{n}\right)$

$\operatorname{MAX}(X)=\operatorname{MAX}\left(X_{1}, X_{2}, \ldots, X_{\text {sid }}, \ldots, X_{n}\right)$

$X_{\text {sid }}^{\prime}=\frac{X_{\text {sid }}-\operatorname{MIN}(X)}{\operatorname{MAX}(X)-\operatorname{MIN}(X)}$

The key issue in calculating sub-CENs from the standardized 15 measured indicators was how to weigh each indicator with minimal subjectivity. The entropy-based weight method as used by other researchers was adopted. In the natural sciences, thermodynamics entropy is used to measure the disorder of a system. In social sciences, information entropy means the uncertainty degree of a system. It is generally believed that the higher information entropy one system has, the more balanced its structure and the smaller its difference. With this view, the information entropy for each index was calculated, and a smaller entropy means a higher weight.

Taking the calculation of NCEN for the sample sid as an example:

Define $N 1_{\text {sid }}^{\prime \prime}$ as the proportion of $N_{\text {sid }}^{\prime}$ in the sample collection, and the numbers of samples is $n$

$N 1_{\text {sid }}^{\prime \prime}=\frac{N 1_{\text {sid }}^{\prime}}{\sum_{i=1}^{n} N 1_{i}^{\prime}}$

Define $e_{N 1}$ as the information entropy of indicator $N 1$ :

$e_{N 1}=-\frac{1}{\ln (n)} \sum_{i=1}^{n}\left(N 1_{i}^{\prime \prime} \times \ln \left(N 1_{i}^{\prime \prime}\right)\right), \quad 0 \leq e_{N 1} \leq 1$
Define $d_{N 1}$ as the redundancy of $e_{N 1}$ :

$d_{N 1}=1-e_{N 1}$

Using the same method as above, $d_{N 2}, d_{N 3}, d_{N 4}$, and $d_{N 5}$ can be calculated.

Because NCEN was made up by $N_{1}, N_{2}, N_{3}, N_{4}$ and $N_{5}$, define $W_{N 1}$ as the weight of

$W_{N 1}=\frac{d_{N 1}}{d_{N 1}+d_{N 2}+d_{N 3}+d_{N 4}+d_{N 5}}$

Define $N 1_{\text {sid }}^{\prime \prime \prime}$ as sid's value under indicator $N 1$ :

$N 1_{\text {sid }}^{\prime \prime \prime}=W_{N 1} \times N 1_{\text {sid }}^{\prime}$

$N 2_{\text {sid }}^{\prime \prime \prime}, N 3_{\text {sid }}^{\prime \prime \prime}, N 4_{\text {sid }}^{\prime \prime \prime}$, and $N 5_{\text {sid }}^{\prime \prime \prime}$ can be calculated with the same method.

Finally, the value of the sid indicator under NCEN is:

$N C E N_{\text {sid }}=N 1_{\text {sid }}^{\prime \prime \prime}+N 2_{\text {sid }}^{\prime \prime \prime}+N 3_{\text {sid }}^{\prime \prime \prime}+N 4_{\text {sid }}^{\prime \prime \prime}+N 5_{\text {sid }}^{\prime \prime \prime}$

With the same calculation method above, $S C E N_{\text {sid }}$ and $E C E N_{\text {sid }}$ can be calculated.

\subsection{UPA-CEN relationship simulation}

The complexity of the UPA-CEN relationship was tested before it was simulated. An increase in UPS does not necessarily lead to an increase in UPA. The former can be realized by various means that do not reflect an individual's interests, such as the merging of administrative regions, or the upgrading of an administrative unit from rural to urban status. Therefore, because the project focused on the UPA and its complexity, the way the UPA differs from the UPS

Table 1

Index for urban population complex ecological niche.

\begin{tabular}{|c|c|c|c|}
\hline CEN (first level) & Sub-CENs (second level) & Indicators (third level) & Measuring variables ${ }^{\mathrm{a}}$ (fourth level) \\
\hline $\begin{array}{l}\text { Complex } \\
\text { Ecological niche } \\
\text { of Urban } \\
\text { Population } \\
\text { (CEN) }\end{array}$ & $\begin{array}{l}\text { Natural environmental } \\
\text { complex ecological } \\
\text { niche (NCEN) }\end{array}$ & $\begin{array}{l}\text { Air condition (N1) } \\
\text { Water condition (N2) } \\
\text { Green land (N3) } \\
\text { Environmental diversity (N4) } \\
\text { Residential environment (N5) } \\
\text { Education guarantee (S1) } \\
\text { Health guarantee (S2) } \\
\text { Population quality (S3) } \\
\text { Cultural communication (S4) } \\
\text { Entertainment sets (S5) } \\
\text { Work income (E1) } \\
\text { Production efficiency (E2) } \\
\text { Economic openness (E3) } \\
\text { Financial viability (E4) } \\
\text { Infrastructure construction (E5) }\end{array}$ & $\begin{array}{l}\text { Industrial } \mathrm{SO}_{2} \text { emissions intensity }\left(\mathrm{t} / \mathrm{km}^{2}\right)^{\mathrm{b}} \\
\text { Industrial waste water discharge safe rate }(\%)^{\mathrm{b}} \\
\text { Green land rate }(\%) \\
\text { Pervious surface land rate }(\%) \\
\text { Garden area rate }\left(\mathrm{km}^{2} / \mathrm{p}\right) \\
\text { Education expenditure of fiscal expenditure }(\%) \\
\text { Number of doctors per } 10,000 \text { people }\left(\mathrm{p} / 10^{4} \mathrm{p}\right) \\
\text { Number of high school and college students per ten thousand people }\left(\mathrm{p} / 10^{4} \mathrm{p}\right) \\
\text { Traffic passengers number divides population (times) } \\
\text { Number of theaters per } 10,000 \text { people }\left(1 / 10^{4} \mathrm{p}\right) \\
\text { Average work income for one person in a year }(\mathrm{RMB})^{\mathrm{c}} \\
\text { Per person GDP }(\mathrm{RMB}) \\
\text { Percentage of foreign investment in total investment }(\%)^{\mathrm{d}} \\
\text { Number of financial workers per } 10,000 \text { people }\left(\mathrm{p} / 10^{4} \mathrm{p}\right) \\
\text { Road area per person }\left(\mathrm{km}^{2} / \mathrm{p}\right)\end{array}$ \\
\hline
\end{tabular}

\footnotetext{
a All data for the measuring variables' calculations were derived from municipal district data; data from suburbs and counties were not included.

b Reverse index had been taken reciprocal before data calculation and analysis.

c Currency index had been counted in 2011 price with CPI.

d Currency index had been counted in 2011 price with CPI and annual average exchange rate.
} 
is discussed first. To test this contrast, correlations between UPS and urban GDP were examined by both the Pearson's and Spearman's rank procedures. Then, two regression analyses were made and compared between UPS-UPA relationship and the UPS-urban GDP relationship. If both the relationships are significant but the regression for UPS-UPA is not as significant as UPS-urban GDP, it suggests that the determinants of UPA are more complex than the urban economic scale, which decides the UPS. Both correlation analysis and regression analysis were executed using SPSS19 (software), and the data of 285 prefecture-level cities in China in the year 2011 were from the China City Statistical Yearbook (2012). Subsequently, multicollinearity analysis was conducted among 15 indicators and UPA indicators using standardized data. A weak multicollinearity implies a weak displacement, which suggests that every indicator in this CEN indicator system is necessary.

Once the UPA-CEN relationship was found to be a complicated nonlinear one, artificial neural network (ANN) analysis was then used to simulate this relationship. An artificial neural network is a computational simulation of a biological neural network. These models mimic the real life behavior of neurons and the electrical messages they produce between input, processing and the final output from the brain (Feldman, 1990). BP-ANN is often used in ANN simulation. The abbreviation means "errors backward propagation training ANN". For a desired output, the network learns from many inputs. It is a supervised learning method, and is a generalization of the delta rule (a kind of learning rule in artificial neural network method)(Klassen et al., 1988). It requires a dataset of the desired output for many inputs, making up the training set. Its learning algorithm can be divided into two phases: propagation and weight update. Each propagation involves the following steps: (1) Forward propagation of a training pattern's input through the neural network in order to generate the propagation's output activations. (2) Backward propagation of the propagation's output activations through the neural network using the training pattern target in order to generate the differential of all output and hidden neurons. Each weight-synapse (a processing parameter during artificial neural network counting)(Nordström and Svensson, 1992) follows the following steps: (1) multiply its output differential and input activation to get the gradient of the weight. (2) Subtract a ratio of the gradient from the weight. This ratio (percentage) influences the speed and quality of learning; it is called the learning rate. The greater the ratio, the faster the neuron trains; the lower the ratio, the more accurate the training is. The sign of the gradient of a weight indicates where the error is increasing; this is why the weight must be updated in the opposite direction. The propagation and weighting are repeated until the performance of the network is satisfactory (McClelland and Rumelhart, 1988).

Both the relationships between the 15 indicators and the UPA (named ANN15) and the relationships between three sub-CENs and UPA (named ANN3) were simulated in this article. Once the ANNs for two relationships are trained and become significant respectively, the ANNs' "black box" (Li et al., 2009a,b) can express the relationships effectively. And the trained ANNs can be used to simulate UPA outputted according to sub-CENs inputted.

The data used in both the multicollinearity test and the ANN simulation were 220 prefecture-level cities of which all data were valid from the year 2000 to 2011 in China, a total of 2640 samples over 12 years. Comparing with the former relative analysis in the first paragraph of Section 2.4, more various data were needed in the ANN analysis, and this decreased the number of effective sample cities from 285 to 220 . All the data came from China City Statistical Yearbooks (2001-2012). During the ANN training process, the neural network was set in a two-layer structure. The first layer used a tan function as the transfer function with 20 neurons. There is no standard method to choice the structure of ANN, but once the chosen ANN performs well in Adjusted $R^{2}$, it will be considered
Table 2

Correlations UPS vs. UPA and Urban GDP vs. UPA in China 2011.

\begin{tabular}{lcc}
\hline & Urban population density & Urban GDP \\
\hline Urban population & & \\
Pearson's correlation & $0.209^{* *}$ & $0.848^{* *}$ \\
Spearman's correlation & $0.394^{*}$ & $0.751^{* *}$ \\
Linear regression coefficient & 3.666 & 0.069 \\
$t$-Test & 3.596 & 26.942 \\
Linear regression constant & 99.977 & 65.635 \\
$t$-Test & 7.130 & 11.096 \\
Adjusted $R^{2}$ & 0.040 & 0.718 \\
$N$ & 285 & 285 \\
\hline
\end{tabular}

* Correlation is significant at the 0.05 level (2-tailed).

** Correlation is significant at the 0.01 level (2-tailed).

believable (Nordström and Svensson, 1992). The second layer used a linear function as the transfer function with one neuron. The multicollinearity test was performed with SPSS19 (software), and the ANN was performed with Matlab 2011a (software).

\subsection{Evaluation of the influence and changes}

Partial correlation analysis was used to test the correlation between each sub-CEN and UPA. Take NCEN for example, when this paper tested the partial correlation between NCEN and UPA, NCEN was set to be the independent variable, both ECEN and SCEN were set to be the control variables, and UPA was the dependent variable.

When the sub-CEN needed to be discussed in depth, the ANN sensitivity analysis method was used. SCEN and ECEN were kept with their original data, but each of the NCEN data needed to add its own standard deviation. This made up new input data for ANN3, which was trained effectively. ANN3 then stimulated the new output data of UPA. Comparison between the new input NCEN data and the new output UPA data showed the quantitative relation between NCEN and the changes in UPA following each NCEN unit change.

In addition, standard deviation (SD) was set as the measure indicator for the balance among three sub-CENs, as SD is usually set to be the variable of distinctness among three (or more) objects. To provide a general view of the changes during 12 years, special histograms were drawn with the range (both $100 \%$ of the samples and $94 \%$ of the samples with $3 \%$ of the samples excluded at either end of their distribution), average, maximum, and minimum of each sub-CEN and SD for all the samples. The percentage changes and standard deviation were also calculated for each sub-CEN and the results were plotted on a map (Fig. 5(d)).

The data used in this section were 2640 samples in sum over 12 years from China City Statistical Yearbooks (2001-2012). Partial correlation analysis was executed with SPSS19 (software), and ANN sensitivity analysis was performed with Matlab 2011a (software). All the data maps were drawn by Arcgis 10 (software).

\section{Results}

\subsection{The complexity of the influencing factors of UPA}

China is a large country with great geographical spatial differences among its cities. Because of the unbalanced distribution of resources and the starting dates of economic openness, eastern China has a greater population than the western region and has a higher population density (Chu, 2008). However, the disequilibrium of UPS and UPA is not spatially consistent (Fig. 3). A deep analysis with a correlation test (Table 2) suggested a positive relationship between them, but there was no significant 
Table 3

ANN structure and simulation results of CEN indicators to UPA

\begin{tabular}{|c|c|c|c|c|}
\hline \multirow[t]{2}{*}{ ANN structure } & \multicolumn{2}{|c|}{15 indicators-UPA (ANN15) } & \multicolumn{2}{|c|}{3 sub-CENs-UPA (ANN3) } \\
\hline & Number of Neure & Function/Method & Number of Neure & Function/Method \\
\hline Input & 15 & - & 3 & - \\
\hline Hidden layer 1 & 20 & Tangent & 20 & Tangent \\
\hline Hidden layer 2 & 1 & Pureline & 1 & Pureline \\
\hline Output & 1 & - & 1 & - \\
\hline Training & - & Levenberg-Marquardt & - & Levenberg-Marquardt \\
\hline Performance & - & Mean squared error & - & Mean squared error \\
\hline \multirow[t]{2}{*}{ ANN simulation } & \multicolumn{2}{|c|}{15 indicators-UPA (ANN15) } & \multicolumn{2}{|c|}{3 sub-CENs-UPA (ANN3) } \\
\hline & Samples & $\overline{\text { Adjusted } R^{2}}$ & Samples & Adjusted $R^{2}$ \\
\hline Training (70\%) & 1848 & $9.65 e-1$ & 1848 & $8.40 \mathrm{e}-1$ \\
\hline Validation (15\%) & 396 & $9.56 \mathrm{e}-1$ & 396 & $8.52 \mathrm{e}-1$ \\
\hline Testing (15\%) & 396 & $9.26 e-1$ & 396 & $8.13 e-1$ \\
\hline Total $(100 \%)$ & 2640 & $9.59 \mathrm{e}-1$ & 2640 & $8.36 e-1$ \\
\hline
\end{tabular}

linear regression for the correlation. Furthermore, the adjusted $R^{2}$ for the linear regression of urban GDP and UPS (0.718) was much higher than for the UPA and UPS (0.04) (Table 2). This implied a more complex nonlinear relationship between UPA and urban economic power.

A multicollinearity analysis for the 15 indicators in the CEN indicator system resulted in all coefficients' absolute values being less than 0.5 , and all the tolerance values for the 15 indicators were more than 0.4 , of which a value less than 0.1 indicates the existence of multicollinearity (Mansfield and Helms, 1982; Wang et al., 1990). In addition, the maximum of all variance inflation factors was 4.4 , of which the threshold value should be at least 10 to support multicollinearity (Wang et al., 1990; York, 2012). That is to say, there was no multicollinearity relationship among those indicators.

Furthermore, ANN was used to simulate both of the complex nonlinear relationships among the 15 indicators and UPA (named ANN15) and the relationship among the three sub-CENs and UPA (named ANN3). The adjusted $R^{2}$ values (Table 3 ) showed that both ANN15 and ANN3 were trained effectively enough to reflect the actual relationships. Although the adjusted $R^{2}$ values (approximately 0.8 ) in ANN3 were less than those (approximately 0.9) in ANN15, they were still acceptable because there is always lost information when an indicator system is reduced to a multidimensional indicator system. An adjusted $R^{2}$ value more than 0.8 is good enough to prove its effectiveness (Li et al., 2009a,b).
Table 4

Partial correlation analysis among sub-CENs and urban population agglomeration.

\begin{tabular}{lccc}
\hline & NCEN & SCEN & ECEN \\
\hline ANN output UPA & & & \\
Partial Correlation & $-0.896^{* *}$ & $0.072^{* *}$ & $0.050^{* *}$ \\
Sig. (2-tailed) & 0.000 & 0.000 & 0.011 \\
Actual UPA & & & \\
Partial Correlation & $-0.710^{* *}$ & $0.043^{* *}$ & 0.023 \\
Sig. (2-tailed) & 0.000 & 0.028 & 0.247 \\
df. & 2636 & 2636 & 2636 \\
\hline
\end{tabular}

* Correlation is significant at the 0.05 level (2-tailed).

** Correlation is significant at the 0.01 level (2-tailed).

\subsection{The driving influence among UPA and sub-CENs}

Results of a partial correlation analysis among three sub-CENs and UPA (actual UPA and ANN simulation output UPA) (Table 4) showed that NCEN had a significant negative influence on UPA, which is opposite to the common opinion but is supported by a few authors from China (Liu et al., 2007). SCEN had a significant positive influence on UPA, which is in line with the common opinions. ECEN had an insignificant positive influence on UPA (Liu et al., 2007; Raskin, 1995).

Because the empirical analysis results produced an unexpected finding for NCEN and because there was less discussion about the
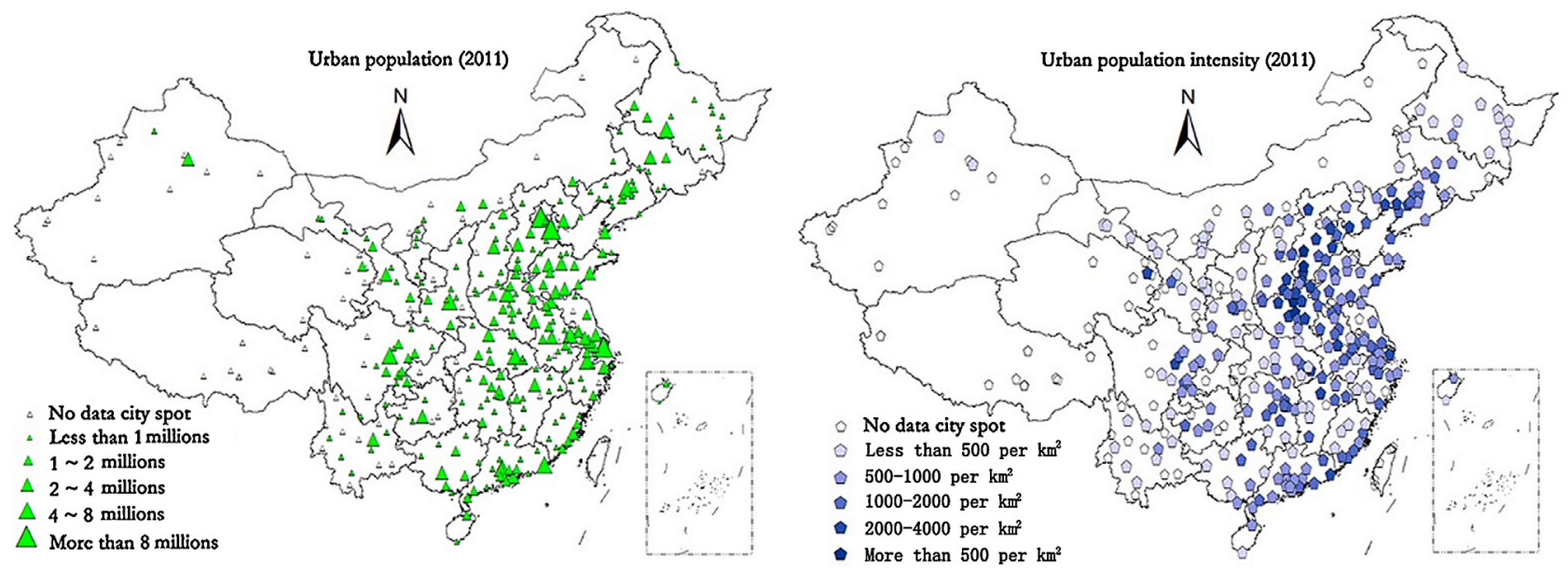


including cities missing data. The left map shows UPS distribution, and UPA is shown on the right. 
(a) Changes of NCEN

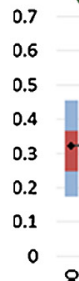

(b) Changes of SCEN

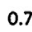

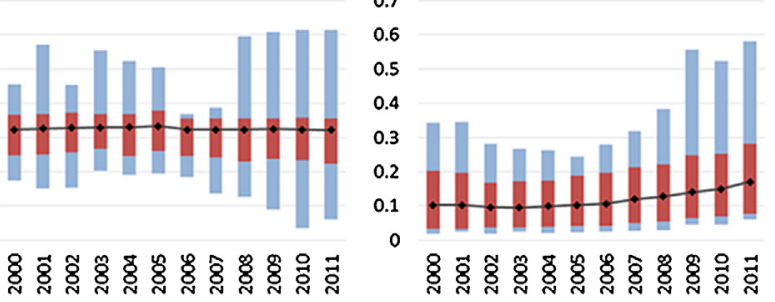

(c) Changes of ECEN

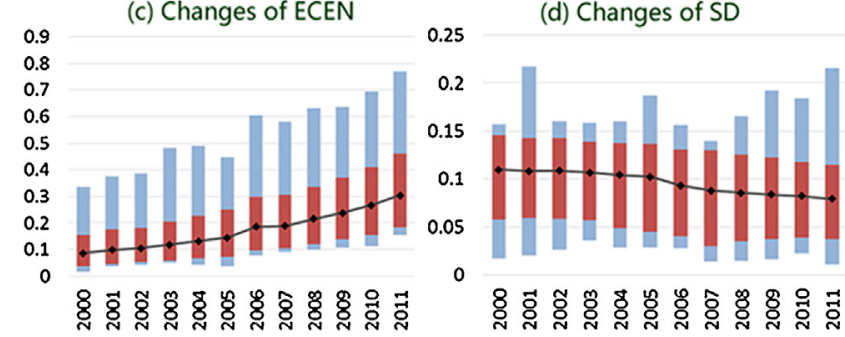

$\square 94 \%$ samples range

$3 \%$ samples range for each side

$\rightarrow$ Average

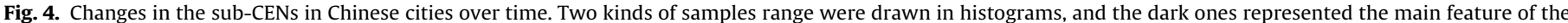
data, in which they contained $94 \%$ of all samples with $3 \%$ excluded on both the largest and smallest parts.

relationship between the urban population density and the urban environment, an in-depth discussion is set out in Section 4.2.

\subsection{Features of the changes on sub-CENs}

During the 2000 to 2011 period, the ranges of all sub-CENs continuously expanded (Fig. 4), implying that the ranges of all subCENs continuously expanded in a view of all samples included, which implied that the diversity of sub-CENs increased and their width expanded. Furthermore, limiting the data to 94\% of all the samples with $3 \%$ excluded on each side showed that NCEN (Fig. 4(a)) was almost stable with a minimally decreasing $(-0.4 \%)$ average value; SCEN (Fig. 4(b)) had a slow increasing trend, and an increase (65\% in sum, $4.7 \%$ for each year on average) of the average value; ECEN (Fig. 4(c)) showed a sharp rising trend with a 253\% (12.1\% for each year on average) increase in the average value. At the same time, the standard deviation (SD) among the three sub-CENs (Fig. 4(d)) kept decreasing during the 12 years, which implied an increase on the balance. Furthermore, according to the main $94 \%$ of the samples, the range of SD was narrower in 2011 compared to 2000. This convergence implied that cities in China became more similar with respect to the three sub-CENs.

The data maps in Fig. 5 show the changes in the three subCENs and SD from 2000 to 2011 shows that regions where NCEN (Fig. 5(a)) improved fastest included the Yangtze River Delta (the river delta zone which contains few cities) and the Zhujiang River Delta. Regions where NCEN was considerably reduced were mainly in the Hebei and Henan Plain areas. SCEN (Fig. 5(b)) in the eastern coastal cities and neighboring provinces increased faster, but in Henan province, degradation was more obvious. ECEN (Fig. 5(c))
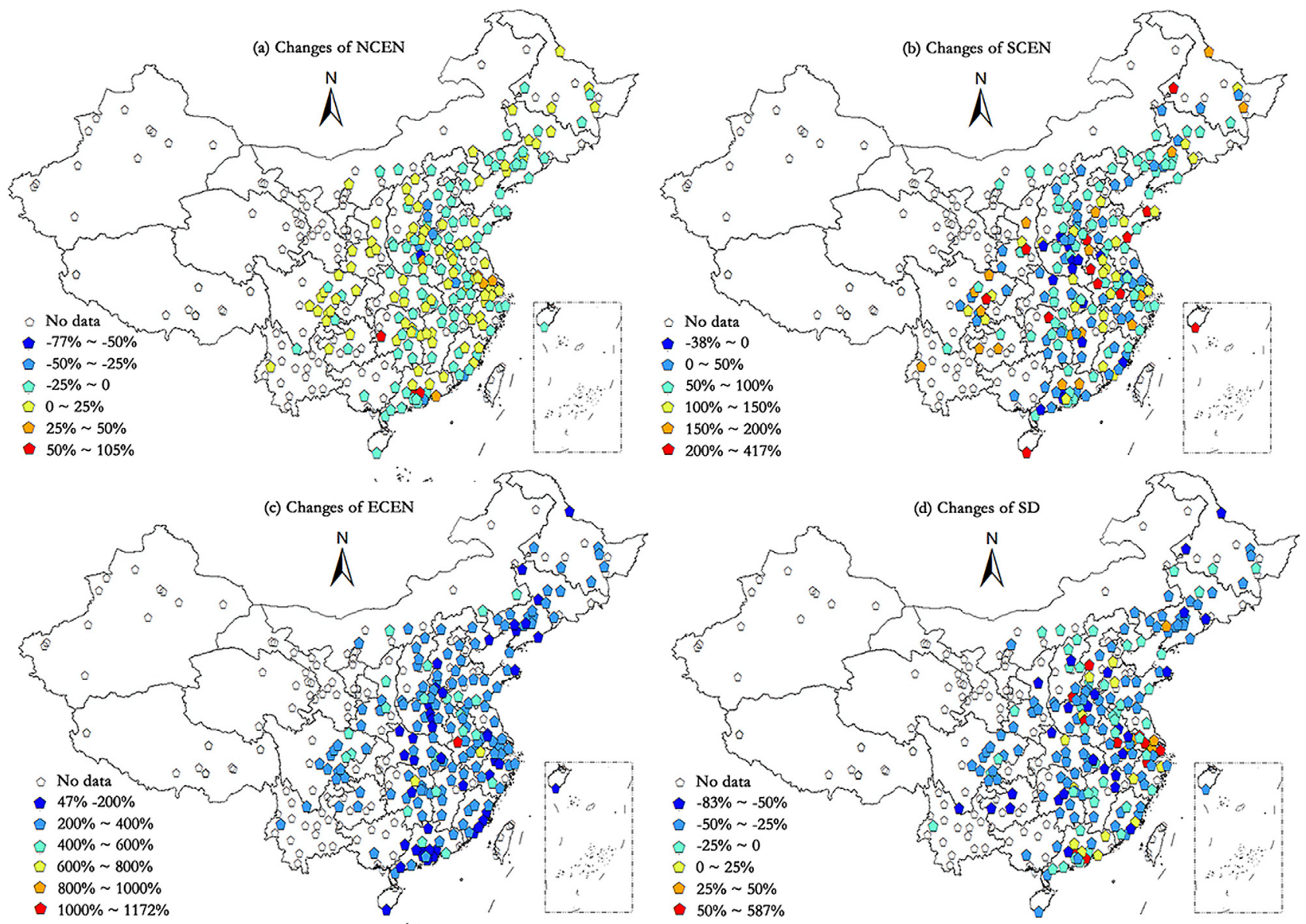

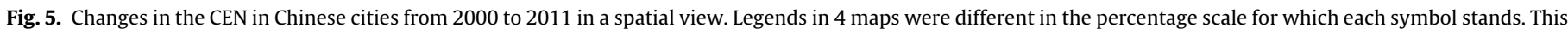
is because the changes of sub-CENs were so different to each other. 
showed a growth trend all over the country, but cities with firmer economic foundations on the east coast had a smaller percentage increase than other cities. The balance of the three sub-CENs (the reciprocal of the SD) showed that all the cities in China became more balanced over the past 12 years except for certain cities in the Yangtze River Delta and the Zhujiang River Delta, where most cities were economically stronger.

\section{Discussion}

\subsection{Explanations for the complexity of CEN for UPA}

The results of the complexity analysis of CEN for UPA can be explained from two standpoints: First, as the urban system is complex, even the 15 measurable indicators chosen in Table 1 demonstrated no multicollinearity. There still would be correlations among the three sub-CENs intuitively, according to other researchers' work (An, 2012; Bennett and McGinnis, 2008; LópezCarr et al., 2012; Wandersee et al., 2012). This is because system theory says "The whole is greater than the sum of the parts". The three sub-CENs of mutual promotion and constraints interact with the development of UPA, and they may follow the cask theory (Zhou et al., 2006) according to the shortest one of the three sub-CENs.

Secondly, although common knowledge says 15 indicators should have a positive effect on UPA, implying that the three subCENs should also have a positive effect on UPA, there is inadequate evidence to support this idea in published research (Song et al., 2008). This project found a negative effect from NCEN on UPA. There is no evidence to support the idea that a worse environment would attract more people. In this view, an explanation with conditioned influence may be suggested, i.e., if there is a low level in one subCEN, it can be compensated by the other sub-CENs. For example, some people prefer more education guarantees over entertainment, and some others may prefer the opposite. If the environment is not good enough to attract people, individuals may pay more attention to other sub-CEN conditions. This implies a threshold value for sub-CENs, which is discussed in Section 4.2.

\subsection{NCEN's conditioned influence on UPA}

As Table 4 showed a significant negative relationship between NCEN and UPA, an in-depth discussion is needed. Because the partial correlation efficient reached a high value (0.896), it was reasonable to test the linear regression function between NCEN and UPA with an overlook of the other sub-CENs. The overlook of ECEN and SCEN did not mean they have nothing to do with UPA but was needed for a clear and direct view of the relationship between NCEN and UPA (Fig. 6). As ANN3 was tested to be effective, the UPA in this section was the simulated output for ANN3. In this view, the functions below could reflect some relationships in ANN3's "black box".

The curve in Fig. 6(a) reflects the relationship of NCEN and UPA; its regression function was

$$
\begin{aligned}
y= & 271.58 x^{6}-695.17 x^{5}+630.51 x^{4}-246.41 x^{3}+39.663 x^{2} \\
& -3.0172 x+0.4938
\end{aligned}
$$

All of the test values for each coefficient were significant (better than the significant level 0.005). The $F$ test for the function was 732.472(sig. 0.000), and the adjusted $R^{2}$ was 0.959 .

The curve in Fig. 6(b) reflects the relationship of NCEN and the changes in UPA per unit change in NCEN; its regression function was

$y=2209.2 x^{5}-3949 x^{4}+2463.4 x^{3}-613.35 x^{2}+50.26 x-1.4909$

All the test values for each coefficient were significant (better than the significant level of 0.005). The $F$ test for the function was 558.337 (sig. 0.000), and the adjusted $R^{2}$ was 0.514 .

Both curves reflect the fact that most of the Chinese cities in this study had a negative relationship between their NCEN and UPA, but the negative effect changed following the change in NCEN There was a threshold (according to Fig. 6(b), which was somewhere between 0.3 and 0.5 ) for the effect; once NCEN passed the threshold, its effect on UPA became positive.

The two explanations for this conditioned influence are (1) Based on common sense, if SCEN and ECEN were invariant when NCEN increased, UPA should increase at the same time. However, the resources used to develop SCEN, ECEN and NCEN were limited, i.e., one sub-CEN cannot increase sharply without a lag in the development of the other sub-CENs. When it came to China's situation during the past 12 years, the development of Chinese SCEN and ECEN was at the cost of natural resources and the environment; thus, the main driving force to UPA increase was SCEN and ECEN, even if their relationships with UPA were not as obvious and as significant as for NCEN. (2) The reason why Chinese people chose a city with a worse NCEN is not because they like the worse environment but because of the substitution effect. As a developing country, both the governors and citizens make development their first goal, which means more money to most people. Each city in China has a plan to develop its manufacturing industry, and they maintain the pollution data as high-rank confidential data. This policy has caused common people to be less sensitive to the environment compared with economic and social guarantees.

In addition, this conditioned influence revealed three key issues: First, the scatter plot in Fig. 6 reflected the fact that people did feel a worsening change in the environment over the past 10 years in China (Yu and Wen, 2010), and people cared more about
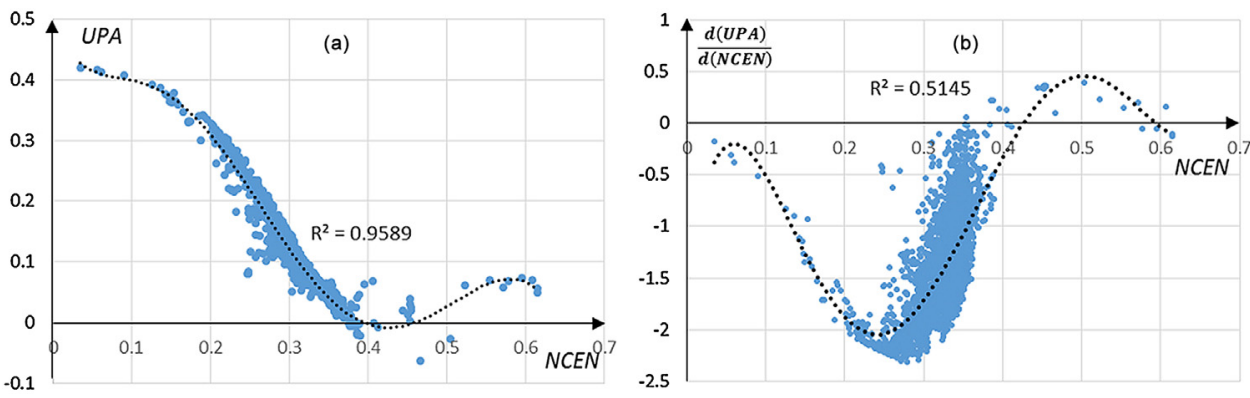

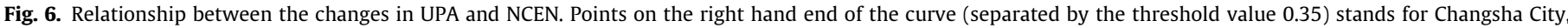


Chinese city. 
their income, food prices and social guarantees than environmental problems. Second, resource allocation imbalance among the three sub-CENs led to the characteristics of an environmental Kuznets curve (Brajer et al., 2011; Song et al., 2008), and most Chinese cities were in the phase where economic development comes at a cost to the environment. Third, there was a weak threshold in Fig. 6, which separated those cities into two types: cities with higher NCEN and a positive relationship with UPA and cities with lower NCEN and a negative relationship with UPA. Once a city's NCEN is around the threshold, the city must make a decisive choice whether they should practice a way to promote UPA with the construction of NCEN or with a cost of NCEN. Although the amount of cities on the right hand of the threshold point is fewer than the left, some strong consistent characteristics among them could be concluded, that is all the cities on the right show a liveable environment and appropriate construction scale for people's daily convenience.

\subsection{Clues for clustering analysis with panel data}

Behind the obvious features in the figures and the significance testing coefficients in the empirical process of this paper, there were still some characteristics of the results which could be used to make this research more accurate. Characteristic 1: the histogram in Fig. 4 reflected that the sample set did not fit a normal distribution; there were characteristics of skew. Although it was not necessary to have a sample set with a normal distribution in the empirical analysis, it revealed the feasibility of classifying the sample set to obtain a few normal distribution sets. Characteristic 2: the scatter plots for both SCEN-UPA and ECEN-UPA reflected nonlinear feature. Although this supports the conclusion that SCEN and ECEN have a positive relationship with UPA, it suggests that classifying the samples by considering their SCEN and ECEN level would be worthful. Clue 3: according to the experience from cities in developed countries, usually there is a division of functions among cities (Futcher et al., 2013); some have a function of production, whereas others, for livelihood and social services, such as entertainment and hospitals (Puissant and Lacour, 2011). Although the division of functions for different cities was not clear in China, it is worth considering such a distinction as a way of classifying them.

\section{Conclusion}

\subsection{Summary}

(1) China's UPA was mainly caused by the individual demand on three aspects: the natural environment, social guarantees, and economic satisfaction. If the range of these factors is viewed as the ecological niche for UPA, the multidimensional space created by the intersection of the various third-level factors makes up the actual CEN for UPA. (2) The effects of NCEN for China's UPA have significant conditioned effect characteristics. SCEN has a positive effect on China's UPA, whereas ECEN also has a positive but weaker effect on China's UPA. (3) In China, NCEN changed little from 2000 to 2011, ECEN had greater progress, and SCEN followed; all three sub-CENs equilibrium levels improved. From the view of spatial characteristics, cities in the Yangtze River Delta and Pearl River Delta had larger enhancements of the natural environmental ecological niches over the 12 years; the improvements in other areas were relatively slow. However, due to the social niche and economic niche of the Yangtze River Delta and Pearl River Delta regions growing much faster than NCEN, the state of equilibrium among the sub-CENs kept degrading. (4) The urban development base has effects on the urban population concentration, but the law has not been identified yet. A follow-up study could use cluster analysis on urban samples from the perspective of SCEN and ECEN to explore various types of urban development and theirs CEN's effect on UPA.

\subsection{Policy suggestions}

(1) The scale of urban development does not reflect the quality of urban development. UPA is affected by the quality of development rather than by the scale of development; therefore, China's urban construction should pay more attention to the per capita value rather than the total volume. (2) The improvement of a city's social facilities and services is the key factor to promote its UPA. Compared to terms of economic factors, social factors are more attractive. China's urban construction should pay more attention to the quality of people's lives rather than the total amount of economic development. Therefore, the priorities within economic development policies in the central and western regions will not necessarily directly improve the CEN of urban population. It would be better to give priority attention to the development of people's livelihood. (3) The ecological environmental construction in the Yangtze River Delta and Pearl River Delta regions since 2000 has had remarkable achievements, but compared to social and economic developments, the NCEN's rate of increase is not fast enough. Governors should further increase investment in ecological environmental construction and ensure that ecological construction at least keeps up with economic development if not progressing even faster. (4) Considering that the positive effects after the NCEN exceeds the threshold, cities with high NCEN and low SCEN should continue to protect and improve their natural environment and develop people's livelihoods. This would be a better and faster way to promote UPA than increasing economic benefits at the cost of the natural environment.

\section{Acknowledgements}

The authors thank Bin Chen, Feng Li, and Liang Yao for their valuable suggestions. This research was supported by National Natural Science Foundation of China (No. 71033005).

\section{References}

An, L., 2012. Modeling human decisions in coupled human and natural systems: review of agent-based models. Ecol. Model. 229, 25-36.

Banks, W.E., d'Errico, F., Peterson, A.T., Vanhaeren, M., Kageyama, M., Sepulchre, P., Ramstein, G., Jost, A., Lunt, D., 2008. Human ecological niches and ranges during the LGM in Europe derived from an application of eco-cultural niche modeling. J. Archaeol. Sci. 35, 481-491.

Bennett, D., McGinnis, D., 2008. Coupled and complex: human-environment interaction in the Greater Yellowstone Ecosystem, USA. Geoforum 39, 833-845.

Brajer, V., Mead, R.W., Xiao, F., 2011. Searching for an Environmental Kuznets Curve in China's air pollution. China Econ. Rev. 22, 383-397.

Chu, T., 2008. Risk factors and genetic markers of human papillomavirus-induced cervical carcinogenesis: a focus on Chinese populations in Southeast Asia and Southern China. Tzu Chi Med. J. 20, 91-100.

Feldman, J.A., 1990. Neural networks, artificial intelligence and computational reality. Comp. Ind. 14, 145-148.

Fujishima, S., 2013. Growth, agglomeration, and urban congestion. J. Econ. Dyn. Control 37, 1168-1181.

Futcher, J.A., Kershaw, T., Mills, G., 2013. Urban form and function as building performance parameters. Building and Environment 62, 112-123.

Gaigné, C., Riou, S., Thisse, J.-F., 2012. Are compact cities environmentally friendly? J. Urban Econ. 72, 123-136.

Grafeneder-Weissteiner, T., Prettner, K., 2013. Agglomeration and demographic change. J. Urban Econ. 74, 1-11.

Gu, C., Wu, L., Ian, C., 2012. Progress in research on Chinese urbanization. Front. Archit. Res. 1, 101-149.

Klassen, M., Pao, Y.-H., Chen, V., 1988. Characteristics of the functional link net: a higher order delta rule net, Neural Networks, 1988. In: IEEE International Conference on IEEE, pp. 507-513.

López-Carr, D., Davis, J., Jankowska, M.M., Grant, L., López-Carr, A.C., Clark, M., 2012. Space versus place in complex human-natural systems: Spatial and multi-level models of tropical land use and cover change (LUCC) in Guatemala. Ecol. Model. 229, 64-75.

Li, C., Guo, S., Zhang, J., 2009a. Modified NLPM-ANN model and its application. J. Hydrol. 378, 137-141.

Li, S., Zhao, Z., Wang, Y., 2009b. Urbanization progress and effects of natural resource and environment in China: research trends and future directions. Prog. Geogr. $28,63-70$. 
Liu, X., Heilig, G.K., Chen, J., Heino, M., 2007. Interactions between economic growth and environmental quality in Shenzhen, China's first special economic zone. Ecol. Econ. 62, 559-570.

Mansfield, E.R., Helms, B.P., 1982. Detecting multicollinearity. Am. Stat. 36, 158-160

McClelland, J.L., Rumelhart, D.E., 1988. An interactive activation model of context effects in letter perception. Part I. An account of basic findings. In: Collins, A., Smith, E.E. (Eds.), Readings in Cognitive Science. Morgan Kaufmann, pp. 580-596.

Munier, N., 2011. Methodology to select a set of urban sustainability indicators to measure the state of the city, and performance assessment. Ecol. Indic. 11, 1020-1026.

Nordström, T., Svensson, B., 1992. Using and designing massively parallel computers for artificial neural networks. J. Parallel Distrib. Comput. 14, 260-285.

Prettner, K., 2013. Population aging and endogenous economic growth. J. Popul. Econ. 26, 811-834.

Puissant, S., Lacour, C., 2011. Mid-sized French cities and their niche competitiveness. Cities 28, 433-443.

Raskin, P.D., 1995. Methods for estimating the population contribution to environmental change. Ecol. Econ. 15, 225-233.

Sato, Y., Yamamoto, K., 2005. Population concentration, urbanization, and demographic transition. J. Urban Econ. 58, 45-61.

Shoshany, M., Goldshlager, N., 2002. Land-use changes in Israel, 1950-1990 and their deriving forces: a comparative two-scale analysis. Land-use Policy 19, 123-133.

Smailes, P.J., Argent, N., Griffin, T.L., 2002. Rural population density: its impact on social and demographic aspects of rural communities. J. Rural Stud. 18, 385-404.

Song, T., Zheng, T., Tong, L., 2008. An empirical test of the environmental Kuznets curve in China: A panel cointegration approach. China Econ. Rev. 19, 381-392.

Tabuchi, T., 2013. Agglomeration in World Cities. Procedia Soc. Behav. Sci. 77, 299-307.

Tabuchi, T., 2014. Historical trends of agglomeration to the capital region and new economic geography. Reg. Sci. Urban Econ. 44, 50-59.
Tan, M., Li, X., Lu, C., Luo, W., Kong, X., Ma, S., 2008. Urban population densities and their policy implications in China. Habitat Int. 32, 471-484.

Tanguay, G.A., Rajaonson, J., Lefebvre, J.-F., Lanoie, P., 2010. Measuring the sustainability of cities: An analysis of the use of local indicators. Ecol. Indic. 10, 407-418.

Theodoridou, I., Papadopoulos, A.M., Hegger, M., 2012. A feasibility evaluation too for sustainable cities-A case study for Greece. Energy Policy 44, 207-216.

Wandersee, S.M., An, L., López-Carr, D., Yang, Y., 2012. Perception and decisions in modeling coupled human and natural systems: A case study from Fanjingshan National Nature Reserve, China. Ecol. Model. 229, 37-49.

Wang, R., 2007. Understanding eco-complexity and promoting ecology of sustainability: Beijing EcoSummit 2007 Review. Acta Ecol. Sin. 27, 2651-2654.

Wang, R., Li, F., Hu, D., Larry Li, B., 2011a. Understanding eco-complexity: socialeconomic-natural complex ecosystem approach. Ecol. Complex 8, 15-29.

Wang, R., Zhou, T., Hu, D., Li, F., Liu, J., 2011b. Cultivating eco-sustainability: social-economic-natural complex ecosystem case studies in China. Ecol. Complex 8, 273-283.

Wang, S., Tse, S., Chow, S., 1990. On the measures of multicollinearity in least squares regression. Stat. Probab. Lett. 9, 347-355.

Wu, J., 2008. Land use changes: economic, social and environmental impacts. Choices $23,6-10$.

York, R., 2012. Residualization is not the answer: rethinking how to address multicollinearity. Soc. Sci. Res. 41, 1379-1386.

Yu, Y., Wen, Z., 2010. Evaluating China's urban environmental sustainability with Data Envelopment Analysis. Ecol. Econ. 69, 1748-1755.

Zhang, L., Zhao, S.X., 2003. Reinterpretation of China's under-urbanization: a systemic perspective. Habitat Int. 27, 459-483.

Zhang, Y., Yang, Z., Yu, X., 2006. Measurement and evaluation of interactions in complex urban ecosystem. Ecol. Model. 196, 77-89.

Zhou, Y., Zhang, J., Zhou, N., Chen, X., 2006. Synthetic assessment with cask theory. Jisuanji Gongcheng/Comp. Eng. 32, 197-199. 